\title{
Research on the Multi-Target Tracking Scheduling Algorithm in the Wire- less Sensor Network
}

\author{
Nie Hai-Yan ${ }^{*}$
}

Zibo Vocational Institute, Zibo, Shan Dong, China

\begin{abstract}
The main problem is how to improve the tracking accuracy and reduce the energy consumption in the multitarget tracking research of the wireless sensor network. The detection extent should be selected according to the relationship between the prediction target state and the sensor nodes. In addition, the selected detection extent can be waked up and then form the clustering set to track the targets. The successful tracking for the multi-targets can be achieved by adjusting the detection extent and adopting time to separate the conflict time in the conflict nodes. The simulation results show that the method proposed in the paper can actually improve the probability of successful tracking.
\end{abstract}

Keywords: Prediction method, wireless sensor network.

\section{INTRODUCTION}

The wireless sensor network is composed of a large number of sensor nodes which are deployed in the specific regions. Each sensor node is equipped with the embedded processor, the sensor component, the storage and the wireless communication. The wireless sensor network can be applied in various fields, such as environmental monitoring, military application, healthy application, household application, business management, target tracking and other fields. Its framework is mainly divided into Sink node and Sensor node. The Sink node mainly collects the data obtained from the environmental monitoring of the sensor nodes. The Sensor node mainly collects the environmental information obtained from the Sensing range.

The Target Tracking in many kinds of environmental monitoring application in the wireless sensor network is always an important application, such as detecting the enemy movements in the military application, tracking the animals' moving routes, nursing the family and others. Tracking the mobile purpose is more difficult than tracking the fixed regions. The target's position information must be updated at any time for obtaining the real-time position information of the target. Therefore, the sensor nodes need to do the localization work to the target at any time and constantly monitor the target's moving method for reducing the chance of losing the target and offering users target information.

\section{RELATED RESEARCHES}

The paper mainly adopts the prediction method to predict the target position. The sensor nodes can be waked up in terms of the prediction target position and the clustering set can be formed to track the target which is the Prediction Based Clustering and the paper does researches aiming to the
Cluster-based target tracking. Many sensor nodes will be formed into the clustering set during the process of the tracking task and then the Cluster Head should be selected from the clustering set, while others belong to the Cluster Member. The cluster member should measure the target information and transmit the information to the cluster head. Later, the cluster head should collect the data, compute and predict the target information and then decide which sensor nodes should do the tracking task in the next time so that the cluster head is very important and energy-consumed. The above description is the Dynamic Clustering framework.

The reference [5-7] introduce an adaptive Sampling interval. The reference [6] proposes a distributed adaptive multi-sensor node scheduling which implements the target tracking with the cooperation of the sensor nodes. The uncertainty of the prediction target position based on the Extended Kalman filter judges whether the uncertain results of the position meets the requirements of the threshold value and then chooses the optimal detection extent. If the sensor nodes can detect the target's probability and set up the probability threshold value during the process of selecting the sensor nodes, $\mathrm{n}$ sensor nodes should be selected to form the clustering set according to the sensor probability and the threshold probability so that the tracking target task can be done. The cluster head can be selected to collect member's information from the clustering set and then the next step's tracking task should be computed and do a signal target tracking.

If the sensor node just can track a target at the same time, the DMTT proposed in the reference [7] can adopt the target's historical route to compute the detection extent. When the target moves in a straight line, the detection extent becomes more larger, while the detection extent becomes more smaller whose target is moving in a irregular line. The EKF should be adopted to predict the target position during the process of choosing the nodes, and the distance between the sensor nodes and the prediction target position should be computed on the basis of Predicted covariance matrix in the Extended Kalman Filter. Then the shortest $\mathrm{n}$ sensor nodes in 
the distance should be selected to form the clustering set and the tracking task should be done. When there are conflict nodes in the multi-target tracking, the conflict sensor nodes will choose which targets should be tracked according to the distance between itself position and the prediction target position and the weighted value among the target importance, and another sensor node can be selected to track another target.

Although the reference [5-7] can choose the detection extent on the basis of the target moving state or the tracking error threshold value, the problem of the node deployment is not considered. If the neighbouring sensor nodes are insufficient during the process of choosing the detection extent, the problems of the target losing and too much localization errors will be caused.

If a target just can be tracked in a tracking task in the multi-target tracking in the reference [7], the problem of having the conflict nodes will be caused with the limitation of the sensor node hard wares. When the conflict nodes happen, the problem of having no nodes to exchange may be caused for it just adopts the Distributed multi-target selection proposed in those above references.

\section{PREDICTION ALGORITHM}

The reference adopts the following three steps to predict the target's next step possible emerging range based on the Prediction Base Target Perdition algorithm: the computation of the present state, the prediction based on the kinematics and the prediction based on the probability.

\subsection{The Computation Of The Present State}

The vectors under the present state of the target in the time point should be computed:

$$
X(k)=(t(k), x(k), y(k), \vec{v}(k), \vec{a}(k))
$$

in which $L(k)=(x(k), y(k))$ is the target's position of the coordinate, $\vec{v}(k)$ and $\vec{a}(k)$ is the target's average speed vector and the target's average acceleration vector within the $(t(k-1), t(k))$.

\subsection{The Prediction Based On The Kinematics}

If the acceleration $\vec{a}(k)$ is not changed from $t(k)$ to $t(k+1)$, its changing rate is the third derivative displacement for the acceleration is the second derivative displacement. The description is usually ignored in the Taylor polynomial formula.

$$
\begin{aligned}
& \overrightarrow{\hat{a}}(k+1)=\vec{a}(k) \\
& \overrightarrow{\hat{v}}(k+1)=\vec{v}(k) \cdot \Delta t \\
& \overrightarrow{\hat{S}}(k+1)=\overrightarrow{\hat{v}}(k+1) \cdot \Delta t+\frac{1}{2} \cdot \overrightarrow{\hat{a}}(k+1) \cdot \Delta t^{2} \\
& \hat{L}(k+1)=L(k)+\overrightarrow{\hat{S}}(k+1)
\end{aligned}
$$

in which $\overrightarrow{\hat{S}}(k+1)$ represents the prediction moving vector from $t(k)$ to $t(k+1)$, and $\overrightarrow{\hat{v}}(k+1)$ represents the prediction position in the $t(k+1)$.

\subsection{The Prediction Based on the Probability}

A probability model can be set up, $S(k+1)$ and $\Delta(k+1)$ are the random variables, that is, $\overrightarrow{\hat{S}}(k+1)=(s(k+1), \Delta(k+1))$.

If $S(k+1)$ is Gauss, that is, $S(k+1) \sim N\left(\mu_{s(k+1)}, \boldsymbol{\sigma}_{s(k+1)}^{2}\right)$. If the acceleration remains unchanging, its computation of average values is as follows:

$\mu_{s(k+1)}=\left\|\overrightarrow{\hat{v}}(k+1) \cdot \Delta t+\frac{1}{2} \cdot \overrightarrow{\hat{a}}(k+1) \cdot \Delta t^{2}\right\|$

in which $\sigma_{s(k+1)}^{2}$ is based on the Gaussian distribution $\ulcorner 68-95-99.7$ rule $\lrcorner$, and $S(k+1)$ man be changed into $\|\vec{v}(k+1) \cdot \Delta t\|$ and $\|\overrightarrow{\hat{v}}(k+1) \cdot \Delta t\|$. Its

probability in the range is about $68 \%$. Therefore, the probability model of the displacement should be set up as follows:

$\sigma_{s(k+1)}^{2}=(\|\hat{S}(k+1)\|-\|\overrightarrow{\hat{v}}(k+1) \cdot \Delta t\|)^{2}$

The target's movement in a short time can be regarded as the linear movement in terms of the inertial property for the detection extent has time limitation so that the angles can be not considered.

\section{MULTI-TARGET TRACKING FRAMEWORK}

If a two-dimensional space in the specific region should be monitored, the sensor nodes should be averagely deployed in the specific regions and then the target tracking task needs to be done. The hard wares in each sensor node is the same, in which their same communicating range is the radius $\mathrm{R}$ and its sensor range radius is $\mathrm{r}$. All sensor nodes can obtain themselves coordinate positions through Global Positioning System or some localization technologies. The sensor nodes can get to know information themselves and their neighboring nodes' information, and those information include the sensor node ID, coordinates and energy. In addition, the sensor node time is synchronous.

The sensor nodes adopts to the low energy consumed communicating channels and those nodes which are not required to be used in the specific regions can be entered the Sleep Mode. The frontier sensor nodes in the specific regions is the sensor mode and the communicating mode. Therefore, 


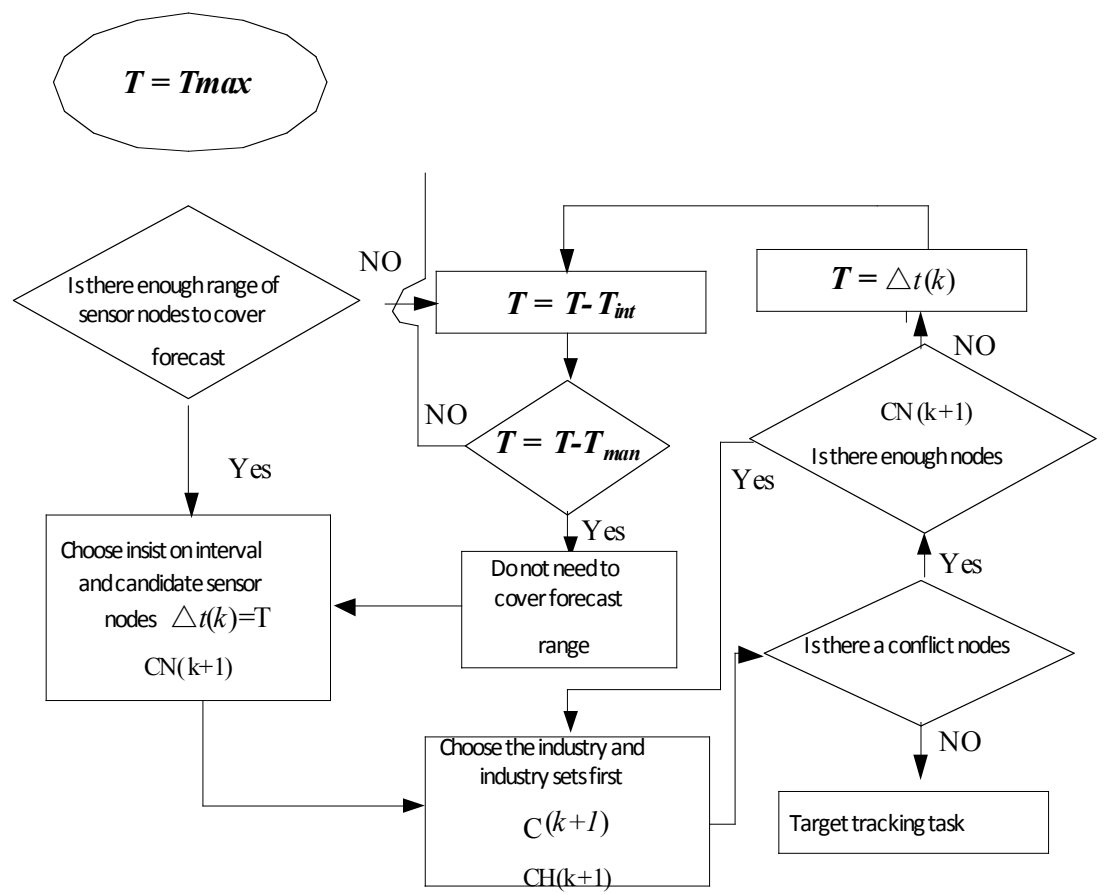

Fig. (1). A complete flow chart of the multi-target tracking algorithm.

if the target should be tracked with the use of these internal sensor nodes, these nodes will be waken up. The specific nodes will form the clustering sets to do the target tracking task. When the task ends, it should enters the Sleep mode. The uncertainty of measuring the target and the false alarms of the sensor nodes can not be considered.

The proposed multi-target tracking algorithm which is regarded the prediction extent scheduling as the prior scheduling is mainly divided into 3 steps:

1. Determine the proper detection extent and the used Candidate Node based on the prediction target state and the position environment of the sensor nodes.

2. The sensor nodes should be selected from the candidate nodes and then the clustering sets can be form. Later, the cluster head can be selected and others are its clustering members. The clustering head can collect the member information and then the next task can be computed.

3. If the conflict nodes happen during the process of tracking the multi-target, other proper sensor nodes or detection extents should be selected for other target and then its tracking sensor nodes can be distributed again.

1. Whether there are enough sensor nodes to cover the prediction range.

2. Whether there are enough nodes.

3. Choose the detection extent and the candidate sensor nodes.

4. Unnecessary to cover the prediction range.

5. Choose out the clustering sets and the cluster head

6. Whether there are conflict nodes.

7. Target tracking task.

\subsection{Choose The Detection Extent $T=T \max$}

The detection extent $(\Delta t(k)=t(k+1)-t(k))$ is an adaptive detection extent and should be adjusted according to each segment's time and the predicted information, and each detection does not use the same detection extent. The selection of the detection extent is limited within the range $\left[T_{\min }, T_{\max }\right], T_{\min } \leq \Delta t \leq T_{\max }$. There are two reasons to limit the size of the detection extent: $T_{\min }$ is the minimum detection extent and it must be larger than the time required by the process of doing the tracking task with the sensor nodes. The tracking task include the following aspects: the sensor time, the transmission delay, the channel storage time and the data processing time. After the current tracking task is done, the system can do the next test. $T_{\max }$ is the maximum detection extent and its maximum detection extent can not be too long for a large number of the target movement states are the non-linear movement. When the detection extent is too long, the difference between the prediction target position and the actual target position is very large.

The prediction target displacement based on the PTP may lie in the $\sigma$ range. Without considering the angles, it is on the center of the prediction target coordinate, in which $\sigma$ is the radius, namely, the prediction range. The node clustering which can do the tracking task in the next time should be found out according to the covering range of the sensor radius $\mathrm{r}$ in the sensor node $S_{i}$, as shown in the Fig. (1). Judge whether the number of the node clustering which can do the tracking task can meet the requirements specified by the algorithm. 


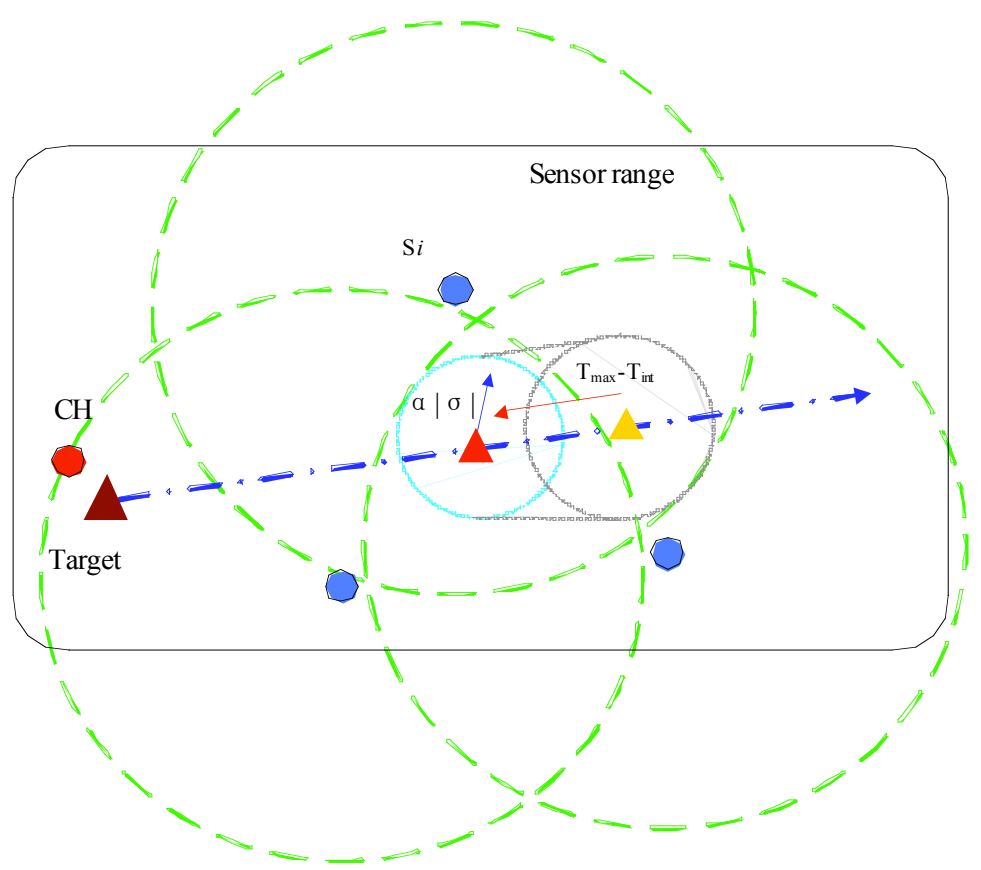

Fig. (2). The figure of the detection extent selection.

The formula can be as follows in terms of computing the prediction range and the sensor displacement position:

$\hat{D}\left(k+1 \mid k, s_{i}\right)+a|\sigma| \leq r$

in which $\hat{D}\left(k+1 \mid k, s_{i}\right)$ is the distance between the prediction target coordinate $\hat{L}(k+1)$ and the sensor node $L s_{i}, \sigma$ is its standard deviation, $\mathrm{r}$ is the sensor radius of the sensor nodes and $a$ is the set-up modulation parameter. The detection extent which meets the requirements of $\mathrm{n}$ sensor nodes can be computed with the use of the judging formula. The algorithm is from the maximum detection extent $T=T_{\max }$ to the $T_{\text {int }}$ until the node clustering which can do the tracking task can meet the requirements of $\mathrm{n}$ node numbers. If the detection extent which meets the requirements of n node numbers cannot be selected, $T_{\min }$ can be regarded as its detection extent, and $T_{\min }$ is used to predict the target position $\hat{L}(k+1)$. Later, the nearest $\mathrm{n}$ sensor nodes should be selected and the prediction range can not be covered.

\subsection{The Selection of The Task Sensor Nodes And The Cluster Head}

A group of sensor nodes can be selected to form the clustering sets for doing the tracking task during the time of each target tracking task, and the clustering head should be selected from the clustering sets and others are its members. The detection extent should be selected from $\mathrm{n}$ sensor nodes. If there are $\mathrm{m}$ candidate nodes, there are candidate sensor nodes to do the tracking task. $\mathrm{n}$ sensor nodes should be selected to do the tracking task from $\mathrm{m}$ sensor nodes. A clustering set can be formed by the sensor node.
As there are many implemented tasks during the time of doing the tracking task with the clustering head, the consumed energy is also rather high. Therefore, the residual energy of the sensor nodes should be considered during the time of selecting the clustering head. The equation of selecting the clustering head is as follows:

$$
C H(k+1)=\arg \max _{s_{i}} E_{s_{i}}, s_{i} \in C(k+1)
$$

in which is the residual energy of the sensor nodes.

\subsection{The Distribution Mechanism Of The Conflict Nodes}

If each node just tracks a target during the time of a task, the previous proposed is the processing time required by the sensor nodes do the tracking task. Therefore, each time of doing the tracking task with the sensor nodes is. When there are two or above targets and the same sensor node is adopted, its sensor node can receive two tracking tasks which needs to be done within the same time, and the node is called Conflict Node.

After the clustering head notice the clustering set which does the next tracking task, it should do continue to do other tasks or enter the sleep mode. Therefore, the distribution mechanism which is used to exclude the conflict nodes should be set up when the later task information is transmitted to the conflict node's clustering head.

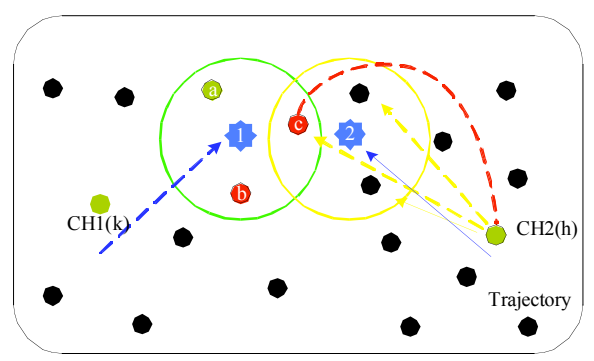

Fig. (3). The distribution mechanism of the conflict node. 
If the sensor node $\mathrm{c}$ in a certain time point receives two tracking task information in the same time, the c node is the conflict node. The conflict node $\mathrm{c}$ will choose the clustering set which is slowly noticed and a conflict packet should be back, including the operating task time, the clustering set information and others. When $\mathrm{CH} 2$ enter the distribution mechanism of the conflict node, the current detection extent should be used to judge whether there are other sensor nodes can be used. If not, the detection extent should be adjusted and the conflict packet information should be delivered. Moreover, the conflict tome segment should be divided with the use of time to reach the purpose of tracking multi targets.

The distribution mechanism of the conflict node can be divided into two parts:

1. The conflict node should be excluded from the candidate node $C N(k+1)$, and then judge whether the sensor nodes are enough. If the candidate nodes are not enough, it should enter the step 2 . If the candidate nodes are enough, the sensor nodes and the clustering head can be selected again.

2. $C H(k)$ can obtain the task time of the conflict nodes and the detection extent can be adjusted in terms of the information. The selected detection extent from the $\mathrm{CH}(k)$ can enter the selection detection extent again and begin to be computed. The detection extent should be computed from $T=\Delta t(k)$ (the conflict node cannot be computed and should be computed after the separation of the conflict time.)

\section{SIMULATION EXPERIMENT}

The proposed multi target tracking algorithm framework should be analyzed with the simulation experiments and be compared with the above proposed DMMT.

\subsection{The Set of The Environmental Simulation}

The simulation adopts Matlab and the sensor nodes should be randomly deployed in the $100 m * 100 m$ range and each sensor node has the same communication radius which is $30 \mathrm{~cm}$, the same sensor radius which is $15 \mathrm{~cm}$ and the same $T_{\text {int }}=0.01$. The detection extent range is limited in the $\left[T_{\max }, T_{\min }\right]=[0.1,0.5]$. If each detection target needs $\mathrm{n}=3$ sensor nodes, the targets can be successfully tested and localized within the three node sensor ranges. The localization model is defined as follows:

$$
\begin{aligned}
& x(k)=t x(k)+v_{x}(k) \\
& y(k)=t y(k)+v_{y}(k)
\end{aligned}
$$

$(t x(k) t y(k))$ is the actual coordinate of the target, $v_{x}(k)$ and $v_{y}(k)$ is a zero mean White Gaussian measurement noise, $\sigma_{x}^{2}=0.001$ and $\sigma_{y}^{2}=0.001$ are the variables.

The deployment density of the sensor node in the Fig. (3) is $1\left(\right.$ node $\left./ 100 \mathrm{~m}^{2}\right)$, the target speed is $10 \mathrm{~m} / \mathrm{s}$ (whose route is $\mathrm{S}$ type) and the adjustable parameter in the prediction range is $a=3$. The Fig. (4) shows the tracking method of the target, and the sensor nodes can be continuously waken up to do the tracking task with the movement of the target.

As the sensor range of the nodes cannot cover the prediction range in the sparse sensor nodes, as shown in the Fig. (4), a smaller detection extent can be selected.

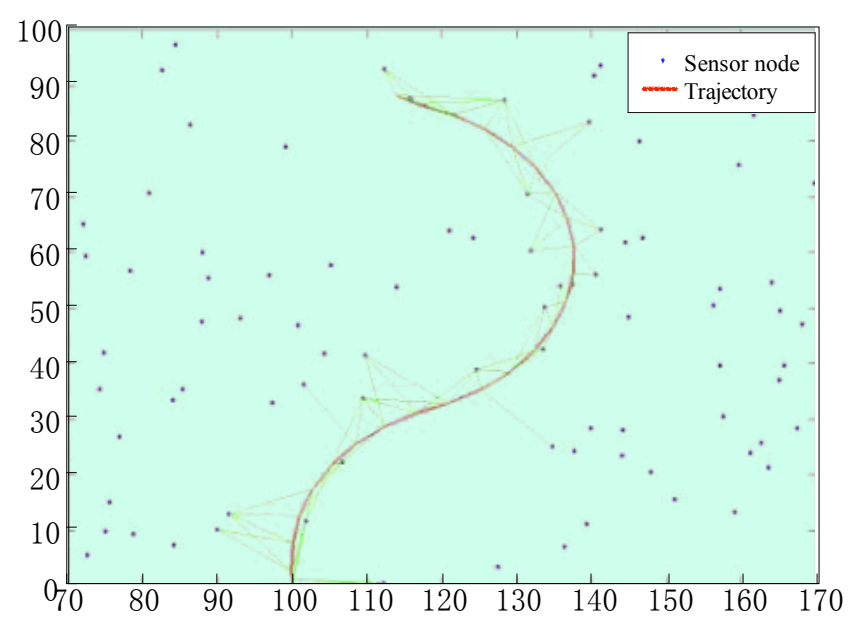

Fig. (4). S type route whose target speed is $10 \mathrm{~m} / \mathrm{s}$.

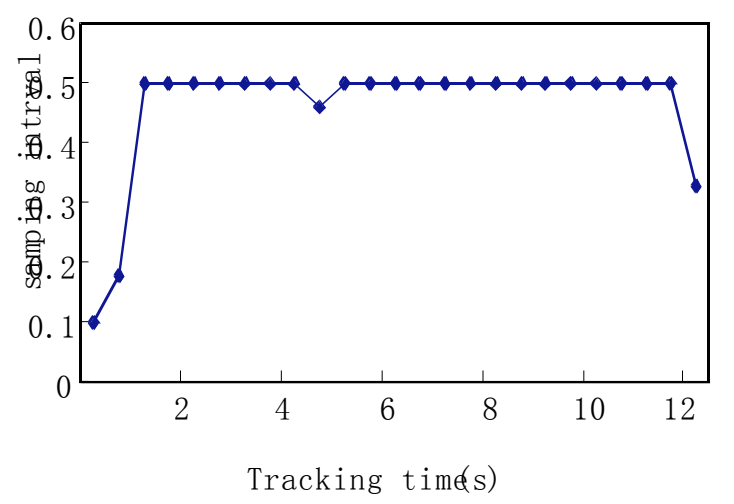

Fig. (5). The change of the detection extent.

If a detection can not meet the requirements of three nodes assuming that each detection target requires three nodes, it is regarded as the fail tracking. When the 100 kinds of sensor nodes ' random distribution are simulated, the deployment density of the three sensor nodes should be compared. The target should have the same speed and the same route movement in the 100 simulations, and the deployment position of the sensor nodes just can be changed.

The Fig. (6) compares the proposed PCF-PTP, DMTT and PCF-EKF. The method PCF-EKF adopts the proposed selected detection extent method and the excluded conflict node method, and its prediction method is EKF [5, 6]. (EKF has no prediction ranges so that it just needs to cover the prediction position.) If the EKF should be set up initially, the target speed with two measurement target positions and the initial values $x(0)$ and $P(0)=10 I$ given by the coordinates should be computed. $I$ is the $4 \times 4$ matrix. 


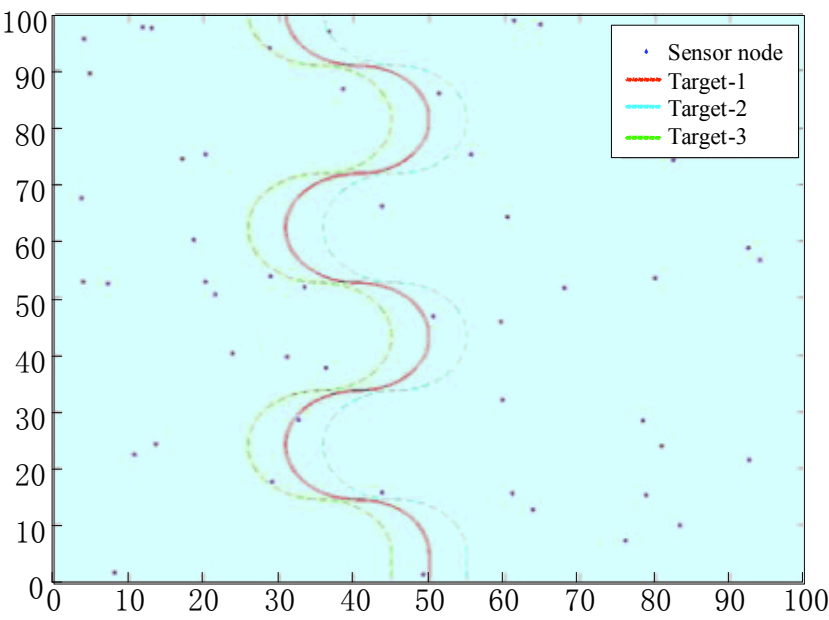

Fig. (5). $\mathrm{S}$ type route of the three targets whose speed is $10 \mathrm{~m} / \mathrm{s}$.

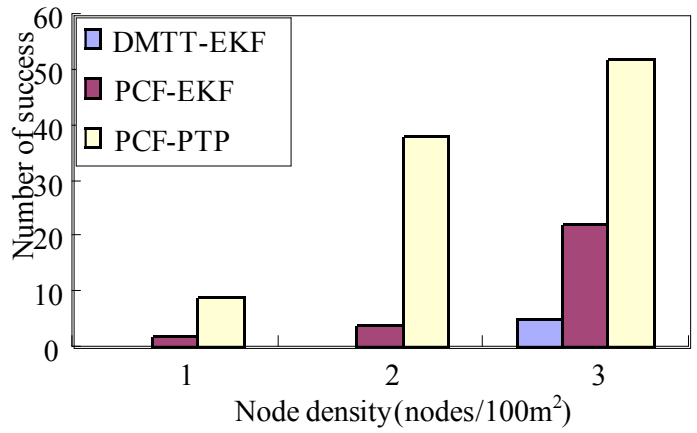

Fig. (6). The tracking successful times in the s type route of the three targets whose speed is $10 \mathrm{~m} / \mathrm{s}$.

\section{CONCLUSION}

The simulations show that the proposed PCF can have a higher tracking successful probability in selecting the detection extent according to the deployment environment of the sensor nodes. The detection extent can be adjusted to exclude the conflict nodes in the sparse sensor nodes and the tracked multi targets can have enough sensor nodes' time point to be detected so that it can improve the probability of successful tracking.

\section{CONFLICT OF INTEREST}

The author confirms that this article content has no conflict of interest.

\section{ACKNOWLEDGEMENTS}

Declared none.

\section{REFERENCES}

[1] K. Ramya, K. Praveen Kumar and Dr. V. Srinivas Rao, "A Survey on Target Tracking Techniques in Wireless Sensor Networks," in Proc. of IJCSES Vol.3, no.4, August 2012.

[2] I. F. Akyildiz, W. Su, Y. Sankarasubramaniam, and E.Cayirci, "Wireless sensor network: A survey," in Proc. of Comput. Netw., vol. 38, no. 4, pp. 393-442, Mar. 2002

[3] T. Malik, M. Sanjay, "Sensor networks: an overview," in Proc. of Potentials, IEEE, vol.22, no.2, pp.20,23, April-May 2003.

[4] M. Fayyaz, "Classification of Object Tracking Techniques in Wireless Sensor Networks" in Proc. of Wireless Sensor Network, vol.3, pp. 121-124, 2011.

[5] W. Xiao, S. Zhang, J. Lin, "Energy-efficient adaptive sensor scheduling for target tracking in wireless sensor networks," in Proc. of J. Control Theory Appli., p. 86-92, 2010.

[6] J. Lin, W. Xiao, F. L. Lewis, and L. Xie, "Energy-efficient distributed adaptive multisensor scheduling for target tracking in wireless sensor networks," in Proc. of IEEE Trans. Intrum. Meas., vol. 58, no. 6, pp. 1886-1897, Jun. 2009

[7] Y. E M. Hamouda and C. Phillips, "Adaptive sampling for energyefficient collaborative multi-target tracking in wireless sensor networks," in Proc. of Wireless Sensor Systems, IET , vol.1, no.1, pp.15-25, March 2011.

[8] Bo Jiang, B. Ravindran, and Hyeonjoong Cho, "Probability-Based Prediction and Sleep Scheduling for Energy-Efficient Target Tracking in Sensor Networks," in Proc. of Mobile Computing, IEEE Transactions on , vol.12, no.4, pp.735-747, April 2013.

[9] Babu, S.S., Raha, A. and Naskar, M.K. A Direct Trust Dependent Link State Routing Protocol Using Route Trusts for WSNs (DTLSRP). Wireless Sensor Network, vol.3, pp.125-134,2011.

[10] Zahariadis, T., Leigou, H.C., Trakadas, P. and Voliotis, S. Mobile Networks: Trust Management in Wireless Sensor Networks. European Transactions on Telecommunications, vol. 21, pp.386-395, 2010.

(C) Nie Hai-Yan; Licensee Bentham Open.

This is an open access article licensed under the terms of the (https://creativecommons.org/licenses/by/4.0/legalcode), which permits unrestricted, noncommercial use, distribution and reproduction in any medium, provided the work is properly cited. 